\title{
Solving the Hubble tension without spoiling big bang nucleosynthesis
}

\author{
Guo-yuan Huang ${ }^{*}$ and Werner Rodejohann $\odot^{\dagger}$ \\ Max-Planck-Institut für Kernphysik, Postfach 103980, D-69029 Heidelberg, Germany
}

(Received 22 February 2021; accepted 6 May 2021; published 7 June 2021)

\begin{abstract}
The Hubble parameter inferred from cosmic microwave background observations is consistently lower than that from local measurements, which could hint towards new physics. Solutions to the Hubble tension typically require a sizable amount of extra radiation $\Delta N_{\text {eff }}$ during recombination. However, the amount of $\Delta N_{\text {eff }}$ in the early Universe is unavoidably constrained by big bang nucleosynthesis (BBN), which causes problems for such solutions. We present a possibility to evade this problem by introducing neutrino selfinteractions via a simple Majoron-like coupling. The scalar is slightly heavier than $1 \mathrm{MeV}$ and allowed to be fully thermalized throughout the BBN era. The rise of neutrino temperature due to the entropy transfer via $\phi \rightarrow \nu \bar{\nu}$ reactions compensates for the effect of a large $\Delta N_{\text {eff }}$ on BBN. Values of $\Delta N_{\text {eff }}$ as large as 0.7 are in this case compatible with BBN. We perform a fit to the parameter space of the model.
\end{abstract}

DOI: 10.1103/PhysRevD.103.123007

\section{INTRODUCTION}

The Hubble parameter inferred from the Planck observations of the cosmic microwave background (CMB), $H_{0}=67.4 \pm 0.5 \mathrm{~km} / \mathrm{s} / \mathrm{Mpc}$ [1], is in tension with that of local measurements at low redshifts. To be specific, the result from the Hubble Space Telescope (HST) by observing the Milky Way cepheids is $H_{0}=74.03 \pm$ $1.42 \mathrm{~km} / \mathrm{s} / \mathrm{Mpc}$ [2], which exceeds the value of Planck experiment by a $4.4 \sigma$ significance. Combining the HST result with a later independent determination [3] yields a lower value of $H_{0}=72.26 \pm 1.19 \mathrm{~km} / \mathrm{s} / \mathrm{Mpc}$, but the tension still persists at $3.7 \sigma$ level.

The tension for the Hubble parameter could suggest the existence of new physics beyond the Standard Model or beyond the $\Lambda \mathrm{CDM}$ framework [4]. A model-independent measure of the impact of new physics can be represented by the effective number of neutrino species $N_{\text {eff }}=3\left(\rho_{\text {rad }}-\rho_{\gamma}\right) /$ $\rho_{\nu}^{\text {std }}$, where $\rho_{\text {rad }}\left(\rho_{\gamma}\right)$ accounts for the energy density of all radiation (photons) and $\rho_{\nu}^{\text {std }}$ for the neutrino energy density in the standard case assuming instantaneous neutrino decoupling. There is a strong positive correlation between $H_{0}$ and an extra radiation, $\Delta N_{\text {eff }} \equiv N_{\text {eff }}-3$, in the early Universe [5]. Hence, by increasing $N_{\text {eff }}$ during recombination, one can lift the Hubble parameter. However, increasing $N_{\text {eff }}$ delays

\footnotetext{
guoyuan.huang@mpi-hd.mpg.de

werner.rodejohann@mpi-hd.mpg.de
}

Published by the American Physical Society under the terms of the Creative Commons Attribution 4.0 International license. Further distribution of this work must maintain attribution to the author(s) and the published article's title, journal citation, and DOI. Funded by SCOAP. matter-radiation equality and modifies the CMB power spectrum. This, in turn, can be compensated by introducing nonstandard neutrino self-interactions during recombination $[6,7]$. Thus, a successful particle physics model to explain the Hubble tension needs to provide a significant amount of $\Delta N_{\text {eff }}$ in the early Universe, as well as "secret" neutrino interactions. In the original fits with Planck 2015 data, two modes of self-interacting neutrinos with the effective coupling form $G_{\text {eff }} \bar{\nu} \nu \bar{\nu} \nu$ are identified, which are given in Table I. The mode with strongly interacting neutrinos $(\mathrm{SI} \nu)$ is basically excluded by various terrestrial experiments [8-12], so we shall confine ourselves to moderately interacting neutrinos $(\mathrm{MI} \nu)$.

Among the many attempts [17-27] (see Ref. [28] for a recent review), one of the simplest possibilities is the Majoron-like interaction [24-27], ${ }^{1}$

$$
\mathcal{L} \supset g_{\alpha \beta} \phi \overline{\nu_{\alpha}} \nu_{\beta},
$$

where $\nu \equiv \nu_{\mathrm{L}}+\nu_{\mathrm{L}}^{\mathrm{c}}, \alpha$, and $\beta$ run over flavors $e, \mu$, and $\tau$, and $g_{\alpha \beta}$ are flavor-dependent coupling constants. After the neutrino temperature drops below $m_{\phi}$, the interactions among neutrinos will be reduced to an effective coupling $G_{\text {eff }} \bar{\nu} \nu \bar{\nu} \nu$ with $G_{\text {eff }}=|g|^{2} / m_{\phi}^{2}$. The flavor-specific couplings $g_{e e}$ and $g_{\mu \mu}$ are severely constrained by laboratory searches [8-12], and we are only left with $g_{\tau \tau}$ to accommodate the MI $\nu$ mode during recombination.

\footnotetext{
${ }^{1}$ This type of coupling may be connected to the neutrino mass generation via the Majoron model [29-36], where both scalar and pseudoscalar couplings exist after the spontaneous breaking of lepton number. For singlet Majorons, it can be generated by mixing with heavy right-handed Majorana neutrinos in a gauge invariant UV completion.
} 
TABLE I. Central values and $1 \sigma$ ranges of two modes in the fit of Planck 2015 data [7]. SI $\nu$ (MI $\nu$ ) stands for the strongly (moderately) interacting neutrino mode; $\eta_{10} \equiv \eta_{\mathrm{b}} \times 10^{10}$ represents the baryon-to-photon ratio. These results are updated with the Planck 2018 data in Refs. [13-16].

\begin{tabular}{lccc}
\hline \hline Parameter & $\log _{10}\left(G_{\text {eff }} \cdot \mathrm{MeV}^{2}\right)$ & $\Delta N_{\text {eff }}$ & $\eta_{10}$ \\
\hline SI $\nu$ & $-1.35_{-0.07}^{+0.12}$ & $1.02 \pm 0.29$ & $6.151_{-0.090}^{+0.079}$ \\
$\mathrm{MI} \nu$ & $-3.90_{-0.93}^{+1.00}$ & $0.79 \pm 0.28$ & $6.253 \pm 0.082$ \\
\hline \hline
\end{tabular}

In our model, the scalar particle $\phi$ with a mass $m_{\phi}$ increases $N_{\text {eff }}$. As long as the coupling in Eq. (1) is strong enough, $\phi$ will be in thermal equilibrium with the neutrino plasma, contributing to extra radiation by $\Delta N_{\text {eff }}=1 / 2$. $8 / 7 \simeq 0.57$ for $m_{\phi} \ll T_{\nu}$, where $T_{\nu}$ is the plasma temperature. Note that $\phi$ is in equilibrium before $\mathrm{BBN}$ as long as $g_{\alpha \beta} \gtrsim 2.2 \times 10^{-10}\left(\mathrm{MeV} / m_{\phi}\right)$ [37]. However, as in many other models, this framework is put under pressure by the primordial element abundances from big bang nucleosynthesis $(\mathrm{BBN})$ [8,37-40]. Incorporating the latest observations, BBN sets a strong constraint on the effective number of neutrino species [41],

$$
N_{\text {eff }}=2.88 \pm 0.27
$$

This can be translated into a $2 \sigma$ upper bound $\Delta N_{\text {eff }}<0.42$, which severely limits the presence of extra radiation to solve the Hubble problem.

In this work, we explore a novel possibility that allows a large $\Delta N_{\text {eff }}$ surpassing the standard BBN constraint in Eq. (2). In our Majoron-like model given in Eq. (1), with $m_{\phi} \gtrsim 1 \mathrm{MeV}$, the scalar particle can stay safely in thermal equilibrium throughout the epoch of BBN, in contrast to concerns in the literature $[8,39,42]$. Namely, since $m_{\phi} \gtrsim 1 \mathrm{MeV}$, the neutrino temperature will increase with respect to the photon one due to $\phi \leftrightarrow \nu+\bar{\nu}$ reactions after the neutrinos have decoupled from the electromagnetic plasma at $T_{\nu}^{\mathrm{dec}} \sim 1 \mathrm{MeV}$. The rise in the neutrino temperature (increasing the neutron burning rate) will cancel the effect caused by a larger $N_{\text {eff }}$ (increasing the expansion rate), such that the final neutron-to-proton ratio $n / p$ remains almost the same as in the standard case.

After a realistic BBN simulation is performed using Eq. (1), we depict the chi-square function $\chi_{\mathrm{BBN}}^{2}$ as a function of the scalar mass $m_{\phi}$ in the upper panel of Fig. 1 (blue curve). This $\chi_{\text {BBN }}^{2}$ includes the latest measurements of the helium-4 mass fraction $\left(Y_{\mathrm{P}}\right)$ [43] and the deuterium abundance $(\mathrm{D} / \mathrm{H})$ [44], as well as various nuclear uncertainties. The dotted red curve represents $\chi_{\text {BBN }}^{2}$ obtained simply with Eq. (2), i.e., without any scalar $\phi$ or rise in neutrino temperature, for the given $\Delta N_{\text {eff }}$. Parameters with $\chi_{\mathrm{BBN}}^{2}>4$ are ruled out at $2 \sigma$ level. It can be observed that a $\Delta N_{\text {eff }}$ value as large as 0.7 is allowed for

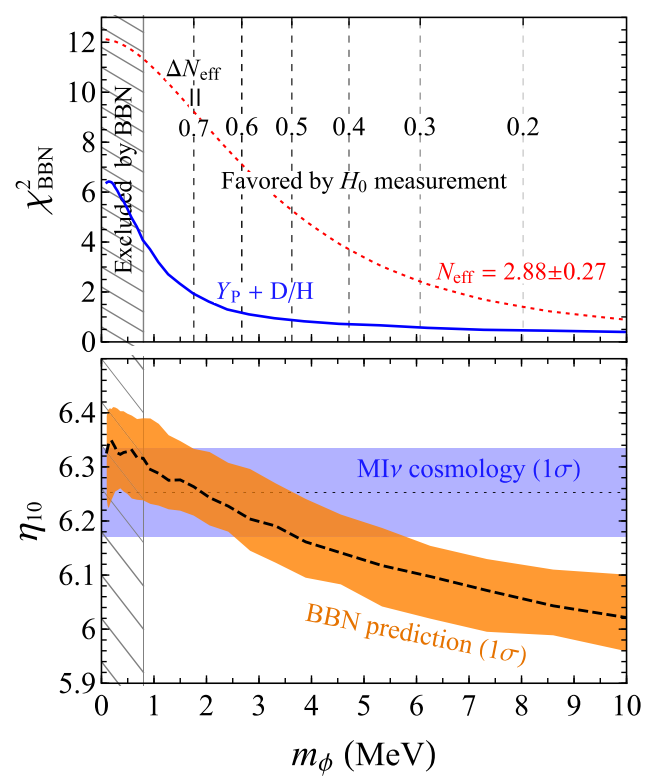

FIG. 1. The statistical significance of $\mathrm{BBN} \chi_{\mathrm{BBN}}^{2}$ (upper panel) and baryon-to-photon ratio $\eta_{10}$ (lower panel) as functions of the scalar mass $m_{\phi}$, assuming $\phi$ is tightly coupled to all three active neutrinos throughout BBN. In the upper panel, the solid blue (or dotted red) curve shows $\chi_{\mathrm{BBN}}^{2}$ by fully simulating nucleosynthesis with the AlterBBN code $[45,46]$ (or adopting the usual bound $N_{\text {eff }}=2.88 \pm 0.27$ [41]). The dashed vertical lines stand for values of $\Delta N_{\text {eff }}$ for corresponding $m_{\phi}$. A value $\Delta N_{\text {eff }} \simeq 0.7$ with $m_{\phi} \gtrsim 1 \mathrm{MeV}$ is permitted by $\mathrm{BBN}$ observations, $\chi_{\mathrm{BBN}}^{2} \simeq 2$, in contrast to the usual BBN bound $\Delta N_{\text {eff }} \lesssim 0.42$ at $2 \sigma$ level [41]. In the lower panel, the yellow region signifies the $1 \sigma$ allowed range of $\eta_{10}$ predicted by helium- 4 and deuterium abundances for different $m_{\phi}$. The independent preferred range given by CMB fit [7] with moderately self-interacting neutrinos is shown in the horizontal blue band. It can be noticed that $m_{\phi} \simeq 2 \mathrm{MeV}$ provides excellent fits to both $\mathrm{BBN}$ and $\mathrm{CMB}$ with $\mathrm{MI} \nu$.

$m_{\phi}=1.8 \mathrm{MeV}$ without spoiling $\mathrm{BBN}$, i.e., $\chi_{\mathrm{BBN}}^{2} \simeq 2$, in contrast to $\chi_{\mathrm{BBN}}^{2} \simeq 9$ using simply the $N_{\text {eff }}$ value in Eq. (2). In the lower panel of Fig. 1, we also show the preferred baryon-to-photon ratio $\eta_{10} \equiv \eta_{\mathrm{b}} \times 10^{10}$ for each $m_{\phi}$. Interestingly, the $1 \sigma$ band around $m_{\phi}=2 \mathrm{MeV}$ matches very well with the independent determination of $\eta_{10}$ from the CMB fit within the moderately self-interacting neutrino case [7]. In contrast, the standard case, which can be roughly represented by $m_{\phi}=10 \mathrm{MeV} \gg T_{\nu}^{\text {dec }}$, disagrees with the $\mathrm{MI} \nu$ value of $\eta_{10}$ by nearly $2 \sigma$.

In the following, we will illustrate the idea and results in more details.

\section{LARGE EXTRA RADIATION FOR BBN}

The improvements in the measurement of primordial element abundances and cross sections of nuclear reactions have made BBN an accurate test for physics beyond the Standard Model [41,47]. The presence of extra radiation 
during the BBN era will accelerate the freeze-out of neutron-proton conversion, resulting in a larger helium-4 abundance than the prediction of standard theory. In addition, the abundance of deuterium is extremely sensitive to the baryon-to-proton ratio $\eta_{\mathrm{b}}$, leaving $\mathrm{BBN}$ essentially parameter-free.

The model-independent bounds as in Eq. (2) are usually applicable to a "decoupled" $\Delta N_{\text {eff }}$, which is assumed to evolve separately from the Standard Model plasma. For the decoupled $\Delta N_{\text {eff }}$, the main effect is to change the expansion rate of the Universe, while leaving other ingredients untouched. However, this is not the case if $\phi$ tightly couples to neutrinos, such that entropy exchange can take place between them. In our case, the argument based on $\Delta N_{\text {eff }}$ should be taken with caution, and we need to solve the primordial abundances.

Two steps are necessary to derive the light element abundances. First, the background evolution of various species $\left(e^{ \pm}, \gamma, \nu\right.$, and $\left.\phi\right)$ needs to be calculated. Second, we integrate this into a BBN code to numerically simulate the synthesis of elements. To calculate the evolution of background species, we solve the Boltzmann equations including the weak interactions between neutrinos and electrons, so the noninstantaneous decoupling of neutrinos is taken into account. More details can be found in the Appendix. We assume that all three generations of active neutrinos are in thermal equilibrium with $\phi$ before and during BBN, which holds for $g_{\alpha \beta} \gtrsim 2.2 \times 10^{-10}\left(\mathrm{MeV} / m_{\phi}\right)$, such that one temperature $T_{\nu}$ is adequate to capture the statistical property of the neutrino- $\phi$ plasma. This greatly boosts our computation without solving discretized distribution functions.

In Fig. 2, we show the evolution of temperature ratio of neutrino to photon $T_{\nu} / T_{\gamma}$ (upper panel) and $N_{\text {eff }}$ (lower panel) as functions of the photon temperature $T_{\gamma}$. Two beyond-standard-model scenarios are given: one with the tightly coupled Majoron-like scalar with mass $m_{\phi}=$ $2.4 \mathrm{MeV}$ (blue curves) and one with the decoupled $\Delta N_{\text {eff }}=0.57$ (red curves). The standard case with only three active neutrinos is shown as gray curves. Note from the lower panel that both scenarios are excluded if we simply adopt the $\Delta N_{\text {eff }}$ bound, i.e., if we disregard the effect of $\phi$ interactions on BBN. In the upper panel, for the case of $m_{\phi}=2.4 \mathrm{MeV}$, shortly after $T_{\gamma}<m_{\phi}$, the neutrino plasma receives entropy from the massive $\phi$, and its temperature is increased by $4.6 \%$ compared to the standard value. In contrast, for the decoupled $\Delta N_{\text {eff }}$ scenario, the ratio $T_{\nu} / T_{\gamma}$ is barely altered. Hence, different from the decoupled $\Delta N_{\text {eff }}$ scenario, there are two effects for the case $m_{\phi}=2.4 \mathrm{MeV}$ : extra radiation $\Delta N_{\text {eff }}$ and a higher neutrino temperature $T_{\nu}$. If we assume the entropy from $\phi$ is completely transferred to neutrinos, the increased temperature can be calculated by using entropy conservation. Namely, $T_{\nu}^{0}=\left(g_{* s} / g_{* s}^{0}\right)^{1 / 3} T_{\nu}=(1+6.0 \%) T_{\nu}$, with $g_{* s} \equiv 25 / 4$ and $g_{* s}^{0}=21 / 4$ being the entropy degrees of

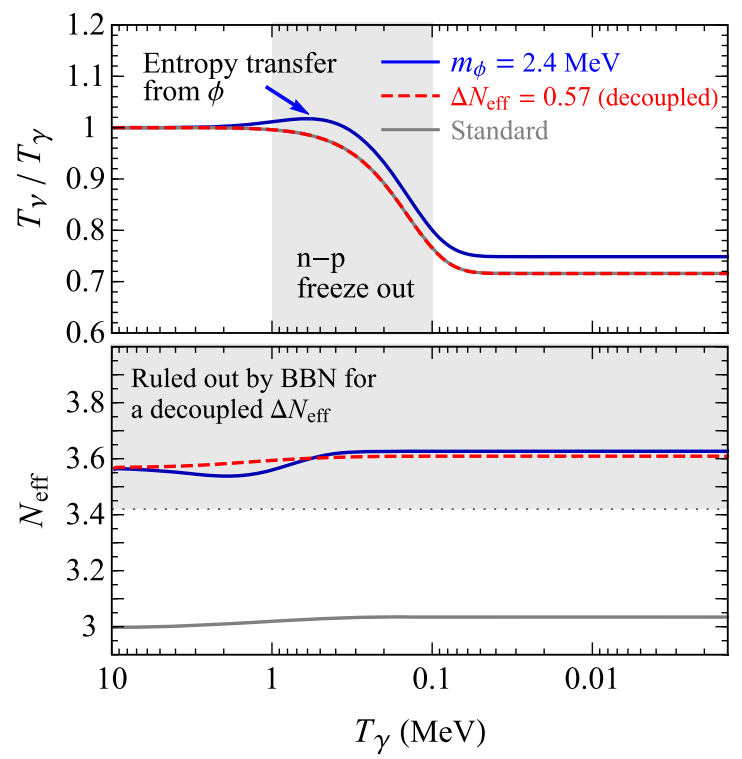

FIG. 2. The temperature ratio for neutrinos and photons $T_{\nu} / T_{\gamma}$ (upper panel) and $N_{\text {eff }}$ (lower panel) with respect to the photon plasma temperature. For all panels, the blue curves stand for the case of $m_{\phi}=2.4 \mathrm{MeV}$ assuming $\phi$ is in thermal equilibrium with neutrinos, while the red curves stand for the case with the decoupled $\Delta N_{\text {eff }}$. The gray curves signifies the standard case with neutrino-electron decoupling taken into account.

freedom before and after $\phi$ decays, respectively. In the realistic case, owing to the weak interactions between neutrinos and electrons, a small part of the entropy goes into the electron-photon plasma.

We now investigate these effects on the neutron-toproton ratio $n / p$, which is the most important $\mathrm{BBN}$ quantity before the deuterium bottleneck at $T_{\gamma} \simeq$ $0.078 \mathrm{MeV}$ is broken through. For the neutron-proton conversion processes where neutrinos appear in the final state, e.g., $\mathrm{p}+e^{-} \rightarrow \mathrm{n}+\nu_{e}$, the neutrino temperature $T_{\nu}$ is relevant only through the Pauli blocking factor $1-f\left(p_{\nu_{e}}\right)$, which is insensitive to the small change of $T_{\nu}$. Here, $f\left(p_{\nu_{e}}\right)$ stands for the Fermi-Dirac distribution function $f\left(p_{\nu_{e}}\right)=1 /\left(1+\mathrm{e}^{p_{\nu_{e}} / T_{\nu}}\right)$, where $p_{\nu_{e}}$ is the momentum of $\nu_{e}$ in the plasma. Thus, we should be concerned about only two processes: $\mathrm{n}+\nu_{e} \rightarrow \mathrm{p}+e^{-}$ and $\mathrm{p}+\overline{\nu_{e}} \rightarrow \mathrm{n}+e^{+}$. The rates are [48]

$$
\begin{aligned}
\Gamma_{\mathrm{n} \nu_{e}}= & \frac{1}{\tau_{\mathrm{n}} \lambda m_{e}^{5}} \int_{0}^{\infty} \mathrm{d} p_{\nu_{e}} \sqrt{\left(p_{\nu_{e}}+Q\right)^{2}-m_{e}^{2}} \\
& \times \frac{p_{\nu_{e}}+Q}{1+\mathrm{e}^{-\left(p_{\nu_{e}}+Q\right) / T_{\gamma}}} \cdot \frac{p_{\nu_{e}}^{2}}{1+\mathrm{e}^{p_{\nu_{e}} / T_{\nu}}}, \\
\Gamma_{\mathrm{p} \overline{\nu_{e}}}= & \frac{1}{\tau_{\mathrm{n}} \lambda m_{e}^{5}} \int_{Q+m_{e}}^{\infty} \mathrm{d} p_{\overline{\nu_{e}}} \sqrt{\left(p_{\overline{\nu_{e}}}-Q\right)^{2}-m_{e}^{2}} \\
& \times \frac{p_{\overline{\nu_{e}}}-Q}{1+\mathrm{e}^{-\left(p_{\overline{\nu_{e}}}-Q\right) / T_{\gamma}}} \cdot \frac{p_{\overline{\nu_{e}}}^{2}}{1+\mathrm{e}^{p_{\overline{\nu_{e}}} / T_{\nu}}},
\end{aligned}
$$


where $m_{e}$ is the electron mass, $Q \equiv m_{\mathrm{n}}-m_{\mathrm{p}} \simeq 1.293 \mathrm{MeV}$, $\lambda \simeq 1.636$, and $\tau_{n}$ the neutron lifetime. When $T_{\nu}<Q$, the rate for $\mathrm{p}+\overline{\nu_{e}} \rightarrow \mathrm{n}+e^{+}$is suppressed by a Boltzmann factor $\mathrm{e}^{-Q / T_{\nu}}$; i.e., only neutrinos with enough initial energy are kinematically allowed for the process. In contrast, the neutron-burning process $\mathrm{n}+\nu_{e} \rightarrow \mathrm{p}+e^{-}$can take place without the energy threshold. Hence, after the decoupling of weak interactions at $T_{\nu} \sim 1 \mathrm{MeV}$, a higher neutrino temperature compared to the standard case will result in a larger neutron burning rate. By ignoring the electron distribution function in the Pauli blocking factor and expanding the square root by taking $m_{e} / Q \simeq 0.15$ as a small quantity, the rate in Eq. (3) can be well approximated by

$$
\begin{aligned}
\Gamma_{\mathrm{n} \nu_{e}} \simeq & \frac{1}{\tau_{\mathrm{n}} \lambda m_{e}^{5}}\left[\frac{45 \zeta(5)}{2} T_{\nu}^{5}+\frac{7 \pi^{4}}{60} Q T_{\nu}^{4}\right. \\
& \left.+\frac{3}{4}\left(2 Q^{2}-m_{e}^{2}\right) \zeta(3) T_{\nu}^{3}\right] .
\end{aligned}
$$

At low neutrino temperatures, the last term will dominate, i.e., $\Gamma_{\mathrm{n} \nu_{e}} \propto T_{\nu}^{3}$. Consequently, under the small perturbation of the neutrino temperature $\delta T_{\nu}$, the rate will be shifted by $\delta \Gamma / \Gamma_{\mathrm{n} \nu_{e}}=3 \delta T_{\nu} / T_{\nu}$. For the case of $m_{\phi}=2.4 \mathrm{MeV}$ in Fig. 2, the relative temperature shift is about $\delta T_{\nu} / T_{\nu}=4.6 \%$, so we have $\delta \Gamma / \Gamma_{\mathrm{n} \nu_{e}} \simeq 13.8 \%$. During the temperature window $0.2 \mathrm{MeV} \lesssim T_{\nu} \lesssim 1 \mathrm{MeV}$, the total neutron conversion rate $\Gamma_{\mathrm{n}}^{\text {tot }}$ is mainly composed of two processes with similar rates, namely $\mathrm{n}+\nu_{e} \rightarrow \mathrm{p}+e^{-}$and $\mathrm{n}+e^{+} \rightarrow \mathrm{p}+\overline{\nu_{e}}$, so we further have $\delta \Gamma / \Gamma_{\mathrm{n}}^{\text {tot }} \simeq 6.9 \%$. To conclude, for the case with $m_{\phi}=2.4 \mathrm{MeV}$, the change of neutrino temperature induced by the entropy transfer from $\phi$ will increase the total conversion rate from neutrons to protons by almost $6.9 \%$.

The above result will compensate the larger expansion rate caused by a positive $\Delta N_{\text {eff }}$. To see that, let us estimate more precisely the impact of $\Delta N_{\text {eff }}$, through the so-called clock effect [49-51]. Before neutrino decoupling, the Hubble expansion rate is governed by

$$
H \simeq \frac{1.66 \sqrt{g_{*}} T_{\gamma}^{2}}{M_{\mathrm{Pl}}}
$$

where $g_{*}=5.5+7 / 4 \cdot N_{\text {eff }}$ stands for the relativistic degrees of freedom before the annihilation of electrons, and $M_{\mathrm{Pl}}=1.221 \times 10^{19} \mathrm{GeV}$ for the Planck mass. Note that the timescales as $t \propto H^{-1}$. Hence, under a perturbation of $\Delta N_{\text {eff }}$, the amount of time over a certain temperature window (e.g., from $T_{\gamma}=1 \mathrm{MeV}$ to $T_{\gamma}=0.078 \mathrm{MeV}$ ) will be changed by $\delta t / t \simeq-7 / 8 \cdot \Delta N_{\text {eff }} / g_{*}^{\text {std }}$ with $g_{*}^{\text {std }}=10.75$ being the degrees of freedom with $N_{\text {eff }}=3.046$. For our case of $m_{\phi}=2.4 \mathrm{MeV}, \Delta N_{\text {eff }}$ is about 0.6 , leading to $\delta t / t \simeq-4.9 \%$. It is crucial that $\delta t / t$ is negative. The evolution of neutron number before $T_{\gamma}=0.1 \mathrm{MeV}$ is described by

$$
\frac{\mathrm{d} n}{\mathrm{~d} t}=-\Gamma_{\mathrm{n}}^{\mathrm{tot}} n+\Gamma_{\mathrm{p}}^{\mathrm{tot}} p
$$

where $n$ and $p$ are the neutron and proton densities, respectively. As has been mentioned before, the conversion rate from proton to neutron $\Gamma_{\mathrm{p}}^{\text {tot }} \simeq \Gamma_{\mathrm{n}}^{\text {tot }} \mathrm{e}^{-Q / T_{\nu}}$ is highly suppressed for $T_{\nu}<Q \simeq 1.293 \mathrm{MeV}$. After the decoupling of weak interactions at $T_{\nu} \sim 1 \mathrm{MeV}$, the conversion from neutrons to protons will dominate the evolution of $n / p$. Thus, the decreased neutron density in a small unit time window $t$ is given by $\Gamma_{\mathrm{n}}^{\mathrm{tot}} t n$, which is sensitive to both the perturbations of conversion rate and time (through the expansion rate). As a result, the larger conversion rate with $\delta \Gamma / \Gamma_{\mathrm{n}}^{\text {tot }} \simeq 6.9 \%$ and the larger Hubble expansion rate with $\delta H / H \simeq 4.9 \%$ (or $\delta t / t \simeq-4.9 \%$ ) will compensate each other.

Having some analytical understanding, we adopt the AlterBBN code $[45,46]$ to calculate the light element abundances, incorporating the background quantities we solved before (see the Appendix). Note that we numerically solve the element abundances including $n / p$ without making approximations as in the previous analytical discussion. We give in the upper panel of Fig. 3 the evolution of $n / p$ with respect to the photon temperature. For illustration, the lower panel indicates the difference of two nonstandard scenarios to the standard one, e.g., $n /\left.p\right|_{\phi}-n /\left.p\right|_{\text {std }}$. One can clearly notice the different impacts of tightly coupled $\phi$

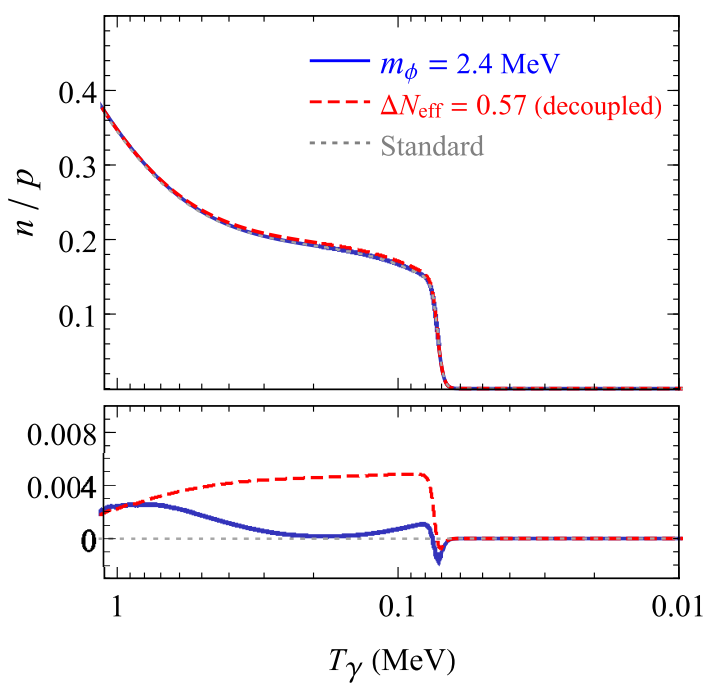

FIG. 3. The neutron-to-proton ratio shown as a function of the photon plasma temperature $T_{\gamma}$ (upper panel). The lower panel gives the differences between the nonstandard scenarios and the standard one. For the cases of $m_{\phi}=2.4 \mathrm{MeV}$ (solid blue curve), the decoupled $\Delta N_{\text {eff }}=0.57$ (dashed red curve) and the standard cosmology (dotted gray curve), the baryon-to-photon ratio has been chosen to minimize the BBN chi-square, namely $\eta_{10}=6.237,6.242$, and 5.997, respectively. The value of $\eta_{10}$ affects the behavior of the curves when the deuterium starts to be copiously synthesized. 
and the decoupled $\Delta N_{\text {eff }}$. For the decoupled $\Delta N_{\text {eff }}, n / p$ takes a larger value than the standard one (by $\sim 0.005$ ) due to the higher expansion rate. This leads to a larger helium-4 abundance by $\delta Y_{\mathrm{p}} \simeq 0.0076$, with $Y_{\mathrm{p}}=2 n /[p(1+n / p)]$, which is significant given the error of the $Y_{\mathrm{p}}$ measurement being about 0.004 [43]. In contrast, for the case of a scalar $m_{\phi}=2.4 \mathrm{MeV}, n / p$ differs from the standard case only by 0.001 , resulting in a negligible change of helium- 4 abundance $\delta Y_{\mathrm{p}} \simeq 0.0015$. In this scenario, $n / p$ increases initially due to the larger expansion rate similar to the decoupled $\Delta N_{\text {eff }}$. Around $T_{\gamma} \simeq 0.9 \mathrm{MeV}$, the increasing neutrino temperature starts to accelerate the burning of neutron, dragging $n / p$ back to the standard value. As a consequence, $Y_{\mathrm{p}}$ is barely altered. This behavior agrees very well with the previous analytical observations. After the deuterium starts to be synthesized around $T_{\gamma}=0.078 \mathrm{MeV}$, the evolution of $n / p$ becomes extremely sensitive to the value of baryon-to-photon ratio $\eta_{10}$. The values of $\eta_{10}$ in Fig. 3 have been chosen to minimize the BBN chi-square, and the given nonstandard cases favor a larger value of $\eta_{10}$ compared to the standard case (as can be observed in Fig. 1). Because the deuterium production rate, i.e., the depletion rate of neutrons and protons, is proportional to $\eta_{10}$, the ratio $n / p$ for new physics scenarios suddenly drops below the standard one near $T_{\gamma}=0.078 \mathrm{MeV}$. Eventually, their differences vanish as $n / p \rightarrow 0$.

\section{PREFERRED PARAMETER SPACE}

Now we explore the preferred parameter space of the model by setting $m_{\phi}$ and $g_{\tau \tau}$ as free parameters, using BBN and other cosmological observations. The primordial values of light element abundances can be inferred from the observation of young astrophysical systems. The mass fraction of helium- 4 has been measured to be $Y_{\mathrm{p}}=$ $0.2449 \pm 0.0040$ by observing the emission spectrum of low-metallicity compact blue galaxies [43]. In addition, the deuterium abundance with a much lower value was derived by observing the absorption spectrum of Lyman- $\alpha$ forests above certain redshifts. The new recommended deuterium-to-hydrogen abundance ratio reads $\mathrm{D} / \mathrm{H}=$ $(2.527 \pm 0.030) \times 10^{-5}$ [44]. On the other hand, the predicted value of $Y_{\mathrm{p}}$ from $\mathrm{BBN}$ is dominated by the neutron-to-proton ratio $n / p$. A standard freeze-out value $n / p \simeq 1 / 7$ with $N_{\text {eff }} \simeq 3$ will give rise to $Y_{\mathrm{p}} \simeq 0.25$. The synthesis of deuterium is more complex, depending on both $N_{\text {eff }}$ and $\eta_{\mathrm{b}}$. The remarkable sensitivity of $\mathrm{D} / \mathrm{H}$ to $\eta_{\mathrm{b}}$ makes it an excellent baryon meter, especially with the recent update of deuterium-related nuclear rates [52]. In the following, $Y_{\mathrm{p}}$ and D/H will be used to constrain our model.

The mass of the scalar $\phi$ cannot be arbitrary in our scenario. If $m_{\phi}$ is too large, the entropy will be mostly released before the neutrino decoupling epoch, and the resulting $\Delta N_{\text {eff }}$ is inadequate to explain the Hubble tension (see also Ref. [53]). In addition, the predicted $\eta_{\mathrm{b}}$ is lower than the value favored by the MI $\nu$ cosmology. On the other hand, if $m_{\phi}$ is too small, there is not enough entropy transfer during the $\mathrm{BBN}$ era, and the $\mathrm{BBN}$ constraint on $\Delta N_{\text {eff }}$ cannot be evaded. This will confine the working range of $m_{\phi}$, as seen in Fig. 1.

To fully explore the parameter space of scalar mass $m_{\phi}$ and coupling constant $g_{\tau \tau}$, we incorporate our results into a fit of $\mathrm{CMB}$ and large scale structure with self-interacting neutrinos. We note that the observational data of CMB and structure formation were initially used to derive bounds on the secret neutrino interactions [54-63], but later a degeneracy was noticed between the effective neutrino coupling $G_{\text {eff }}$ and other cosmological parameters [6], which can help to resolve the Hubble issue. With the Planck 2015 data, the Hubble tension can be firmly addressed by a large $\Delta N_{\text {eff }}$ along with self-interacting neutrinos [7]. However, the fits based on the latest Planck 2018 data (specifically with the high- $l$ polarization data) show no clear preference for strongly interacting neutrinos $[13,14]$. The results omitting the high- $l$ polarization data however remain similar to the analysis with Planck 2015 data. In either case, including the local measurement of $H_{0}$ will always induce a preference for large $\Delta N_{\text {eff }}$ and nonvanishing $G_{\text {eff }}$, but the overall fit with the high- $l$ polarization data of Planck 2018 is poor.

In order to be definite, we will adopt the results where only $\nu_{\tau}$ moderately couples to $\phi$ during the recombination epoch. These include [16]

$$
\begin{aligned}
\log _{10}\left(G_{\text {eff }} \cdot \mathrm{MeV}^{2}\right) & =-3.2_{-1.5}^{+1.3}, \quad N_{\text {eff }}=3.69_{-0.33}^{+0.28}, \\
\eta_{10} & =6.195 \pm 0.099,
\end{aligned}
$$

with the normal neutrino mass ordering. The baryon-tophoton ratio $\eta_{10}$ is converted from $\Omega_{\mathrm{b}} h^{2}$ by using $\eta_{10}=$ $274 \Omega_{\mathrm{b}} h^{2}$ [64]. For each parameter choice of $m_{\phi}$ and $g_{\tau \tau}$, the total $\chi^{2}$ is constructed as a combination of the BBN one $\chi_{\mathrm{BBN}}^{2}$ and those fitted with the central value and symmetrized $1 \sigma$ error in Eq. (8). The preferred region of parameter space is given in Fig. 4. The dark red region signifies the preferred parameter space for $g_{\tau \tau}$ at $90 \%$ confidence level (CL), inside which the dashed curve stands for the $68 \% \mathrm{CL}$ contour and the star represents the best-fit point, i.e., $m_{\phi}=2.8 \mathrm{MeV}$ and $g_{\tau \tau}=0.07$.

Laboratory and astrophysical searches set stringent upper limits on the secret neutrino interactions [9-12,65, 67-75], especially for the coupling strengths of $\nu_{e}$ and $\nu_{\mu}$ with the scalar. When it comes to the recombination epoch, to achieve moderate self-interactions $G_{\text {eff }} \sim 10^{-3} \mathrm{MeV}^{-2}$, we must have sizable $g_{\tau \tau}$. The bound on $g_{\tau \tau}$ from $Z$ decays is shown as the gray band on the top [10]. On the other hand, to have a higher neutron burning rate, $\nu_{e}$ must stay in equilibrium with $\phi$ during the BBN era as we assumed in the previous discussion, which will impose a lower limit on 


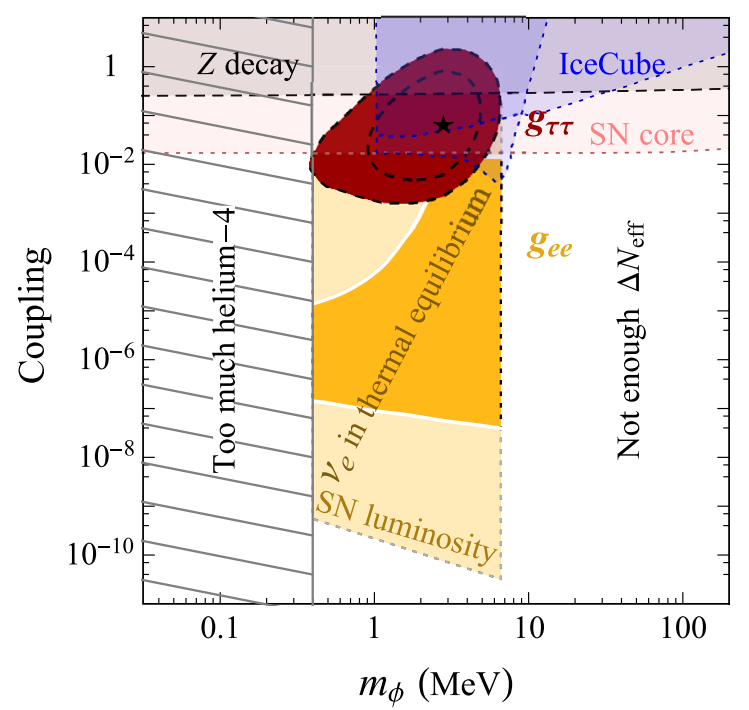

FIG. 4. The scalar coupling versus its mass $m_{\phi}$. The red region is the $90 \%$ preferred parameter space for $g_{\tau \tau}-m_{\phi}$ by taking BBN and fits of CMB and the local value of $H_{0}$ into account [16]. Note that the results of the moderately self-interacting neutrino mode have been used. Inside the red region, the dashed curve surrounds the $1 \sigma$ region, and the star in the middle marks the best-fit point. The bound on $g_{\tau \tau}$ from $Z$ decays is shown in the gray band on the top [10]. The required strength of $g_{e e}$ to keep $\nu_{e}$ and $\phi$ in equilibrium is shown in the yellow region, while various limits to $g_{e e}$ are given as lighter yellow regions $[8,12]$. The modeldependent IceCube limits on the universal couplings are recast as blue curves [65], which should be weakened for the flavorspecific coupling $g_{\tau \tau}$. The supernova limit based on considerations of neutrino-driven shock revival is shown in the pink region [66].

the coupling constant $g_{e e} \gtrsim 2.2 \times 10^{-10}\left(\mathrm{MeV} / m_{\phi}\right)$ [37]. The required coupling strength for $g_{e e}$ is depicted in the yellow region. The lighter yellow regions are excluded by neutrinoless double-beta decay experiments $[12,76]$ (top left), $K$ decays [8] (top right) and supernova luminosity constraint [12] (bottom), respectively. The presence of large $g_{\tau \tau}$ coupling will enhance the invisible decay rate of $Z$ [10], which can be conveniently measured by the number of light neutrino species $N_{\nu}$. Our best-fit case $m_{\phi}=2.8 \mathrm{MeV}$ and $g_{\tau \tau}=0.07$ predicts $N_{\nu}=3.0012$ [10].

The region of our interest for $g_{\tau \tau}$ may lead to a dip in the spectrum of ultra-high energy (UHE) neutrinos observed at IceCube by scattering off the relic neutrinos [65,69-74]. If we assume the neutrino mass to be $m_{\nu}=0.1 \mathrm{eV}$, the resonant-scattering dip should be around $E_{\nu}=m_{\phi}^{2} /\left(2 m_{\nu}\right) \approx 78 \mathrm{TeV}$ for our best-fit case $m_{\phi}=$ 2.8 MeV. The absence of the dip at IceCube will place a constraint on our preferred parameter space [65], recast as dotted blue curves in Fig. 4, which has covered part of our $1 \sigma$ parameter space. However, we need to mention that the actual constraints are subject to the neutrino mass spectrum, the neutrino flavor, the model of sources as well as initial spectrum of UHE neutrinos. For example, in some model where UHE neutrinos are generated from decays of dark matter in Milky Way, the constraints from diffuse spectrum do not apply anymore. The constraints in Ref. [65] will also alter if a different spectrum index or flavor-specific coupling is taken. Another signature of interest is the echo of UHE neutrinos from a transient source induced by the neutrino self-interaction [77], which will be a powerful probe in the future to our inferred parameter region.

Let us emphasize here another astrophysical source that may be sensitive to our favored parameter space. The successful explosion of supernovae and the observation of neutrinos from SN1987A could be affected by the Majoron model [12,66,78-85]. Four types of effects of a MeV-scale Majoron can be found in the literature. First, the deleptonization of the supernova core may prevent the explosion of supernovae. The kinematically allowed process of deleptonization in our scenario is $\nu_{e} \nu_{\alpha} \rightarrow \phi$ with $\alpha$ being $e, \mu$, or $\tau$. Constraints can then be put on the couplings involving the electron flavor based on the deleptonization argument [85]. Second, the Majoron emission should not dominate the loss of the supernova binding energy. The relevant process is $\nu \nu \rightarrow \phi$, which will result in the energy loss by means of $\phi$ emission as long as $\phi$ is not trapped in the core $[12,85]$. This leads to the limit shown as the light yellow region near the bottom of Fig. 4. Third, the energy spectrum of neutrinos from SN1987A can be altered by processes which change the total number of $\nu$ and $\phi$ [78]. Last, the neutrino self-interactions may prevent the shock revival that relies on the neutrino energy deposition and halt the supernova explosion through the process $2 \nu \rightarrow 4 \nu$ [66]. The constraint explored recently based on this consideration is recast as the pink region in Fig. 4. Of course, it is fair to say that these arguments should depend on the valid model of successful supernova explosion. A better understanding of the supernova dynamics will help to reinforce these limits.

\section{CONCLUDING REMARKS}

In this paper, we have explored the role of $\mathrm{BBN}$ for the Majoron-like scalar solution in light of the $H_{0}$ tension. Note that this work is based on scalar interactions of Majorana neutrinos, but similar or slightly modified considerations can also be made for other theories, e.g., complex scalar and vector interactions, and even Dirac neutrinos. By numerically solving the light element abundances, we find that a simple Majoron-like scalar with mass $\gtrsim \mathrm{MeV}$ can provide moderate self-interaction as well as large $\Delta N_{\text {eff }}$ during the recombination epoch to relieve the Hubble tension. By incorporating the latest results of helium-4 and deuterium abundances, $\mathrm{CMB}$ data and local measurements of $H_{0}$, we find that the parameter choice $m_{\phi}=$ $2.8 \mathrm{MeV}$ and $g_{\tau \tau}=0.07$ can provide the best fit. The widely concerned BBN constraint $\Delta N_{\text {eff }} \lesssim 0.42$ does not apply because it ignores the entropy transfer of a MeV-scale 
$\phi$ that heats up the neutrino bath. If we take $m_{\phi}=2.4 \mathrm{MeV}$, the neutrino temperature can be increased by around $4.6 \%$ during the freeze-out of neutron-proton conversion. As a consequence, the neutron burning rate is increased by almost $7 \%$. The extra radiation and the rise in the neutrino temperature are found to compensate each other, such that the $\Delta N_{\text {eff }}$ as large as 0.6 is warranted throughout the BBN era, which should be very helpful to address the $H_{0}$ tension.

\section{ACKNOWLEDGMENTS}

GYH would like to thank Kun-Feng Lyu for inspiring discussions. This work is supported by the Alexander von Humboldt Foundation.

\section{APPENDIX: BACKGROUND EVOLUTION}

In this appendix, we explain how the evolution of the background is obtained in more detail.

In the assumption that three flavors of active neutrinos are all tightly coupled to $\phi$, only one temperature $T_{\nu}$ is sufficient to describe the neutrino- $\phi$ plasma. The state of electron-photon plasma is represented by $T_{\gamma}$. To make the effect of expansion of the Universe explicit, it is convenient to introduce the following dimensionless quantities in the comoving frame:

$$
x \equiv m a, \quad q_{i} \equiv p_{i} \cdot a, \quad z_{i} \equiv T_{i} \cdot a, \quad \tilde{s}_{i}=s_{i} \cdot a^{3},
$$

where $a$ is the dimensionful scale factor, $x$ the dimensionless scale factor with $m$ being an arbitrary mass scale (we set $m=1 \mathrm{MeV}$ ), $q_{i}$ the comoving momentum for species $i$, $z_{i}$ the comoving temperature, and $\tilde{s}_{i}$ the comoving entropy density. If there is only one massless species in the Universe, $q_{i}, z_{i}$ and $\tilde{s}_{i}$ will be constant during the expansion of the Universe.

Taking account the weak interactions, the comoving entropy density transferred from the electron-photon plasma to the neutrino- $\phi$ one can be calculated with $[86,87]$

$H x \frac{\mathrm{d} \tilde{s}_{\nu \phi}}{\mathrm{d} x}=\sum_{\alpha} \int \frac{\mathrm{d}^{3} q}{(2 \pi)^{3}} C_{\nu_{\alpha}}\left[f_{e}, f_{\nu}\right] \ln \left(\frac{f_{\nu_{\alpha}}}{1-f_{\nu_{\alpha}}}\right)$,

where the collision terms $C_{\nu_{\alpha}}\left[f_{e}, f_{\nu}\right]$ for six neutrino flavors $\nu_{\alpha}$ (including antineutrinos) have been widely calculated and are available in the literature, and see, e.g., Refs. [87-89]. When the collision terms are vanishing (i.e., no heating from the electron-photon plasma), the entropy in the neutrino- $\phi$ plasma is conserved. Also note that for the neutrino decoupling process, which is not a thermal equilibrium process, the total entropy $\tilde{s}_{\nu \phi}+\tilde{s}_{e \gamma}$ is not preserved in general. The entropy density of the neutrino- $\phi$ plasma in terms of the neutrino comoving temperature reads $[86,87]$

$\tilde{s}_{\nu \phi}=-\sum_{i=\nu, \phi} \int \frac{\mathrm{d}^{3} q}{(2 \pi)^{3}}\left[f_{i} \ln f_{i} \mp\left(1 \pm f_{i}\right) \ln \left(1 \pm f_{i}\right)\right]$,

where the upper and lower signs apply to bosons and fermions, respectively. Here, the distribution functions for neutrinos and $\phi$ are $f_{\nu}=1 /\left(1+\mathrm{e}^{q_{\nu} / z_{\nu}}\right)$ and $f_{\phi}=1 /\left(1-\mathrm{e}^{\sqrt{q_{\phi}^{2}+x^{2} m_{\phi}^{2} / m^{2}} / z_{\nu}}\right)$, and the sum is over all neutrino species and the $\phi$ boson. We use the following formula to establish the relation between the variation of entropy and that of the neutrino temperature $\mathrm{d} z_{\nu} / \mathrm{d} x$ :

$$
\frac{\mathrm{d} \tilde{s}_{\nu \phi}}{\mathrm{d} x}=\frac{\partial \tilde{s}_{\nu \phi}}{\partial x}+\frac{\partial \tilde{s}_{\nu \phi}}{\partial z_{\nu}} \cdot \frac{\mathrm{d} z_{\nu}}{\mathrm{d} x},
$$

where $\partial \tilde{s}_{\nu \phi} / \partial x$ and $\partial \tilde{s}_{\nu \phi} / \partial z_{\nu}$ can be straightforwardly obtained with Eq. (A3). Some observations on Eq. (A4) are very helpful. If we assume there is no entropy transferred from other species, i.e., $\mathrm{d} \tilde{s}_{\nu \phi} / \mathrm{d} x=0$, the variation of comoving temperature of neutrinos $\mathrm{d} z_{\nu} / \mathrm{d} x$ will be proportional to $\partial \tilde{s}_{\nu \phi} / \partial x$. For $\tilde{s}_{\nu \phi}$, the only explicit dependence on $x$ is associated with the mass of $\phi$ in $f_{\phi}$; therefore, if $m_{\phi} \ll T_{\nu}, \partial \tilde{s}_{\nu \phi} / \partial x$ will be negligible, and $T_{\nu}$ simply follows the redshift as the Universe expands. The function $\partial \tilde{s}_{\nu \phi} / \partial x$ roughly measures the entropy flow from $\phi$ to neutrinos.

On the other hand, the photon temperature can be derived by utilizing energy conservation $x \mathrm{~d} \rho_{\text {tot }} / \mathrm{d} x=$ $-3\left(\rho_{\text {tot }}+P_{\text {tot }}\right)$, namely,

$$
x \frac{\mathrm{d} T_{\gamma}}{\mathrm{d} x}=\frac{-3\left(\rho_{\text {tot }}+P_{\text {tot }}\right)-x \frac{\mathrm{d} T_{\nu}}{\mathrm{d} x}\left(\frac{\mathrm{d} \rho_{\nu}}{\mathrm{d} T_{\nu}}+\frac{\mathrm{d} \rho_{\phi}}{\mathrm{d} T_{\nu}}\right)}{\frac{\mathrm{d} \rho_{\gamma}}{\mathrm{d} T_{\gamma}}+\frac{\mathrm{d} \rho_{e}}{\mathrm{~d} T_{\gamma}}},
$$

along with $m \mathrm{~d} z_{i} / \mathrm{d} x=x \mathrm{~d} T_{i} / \mathrm{d} x+T_{i}$ for $i=\nu$ and $\gamma$. The temperature of the photon-electron plasma can also be derived by solving the electron collision terms similar to that of neutrinos. But since the energy conservation is a guaranteed result of microscopic processes, these two approaches to obtain the photon temperature are actually equivalent. By combining Eqs. (A2), (A4), and (A5), we are ready to solve the background quantities for any given initial conditions. 
[1] N. Aghanim et al. (Planck Collaboration), Planck 2018 results. VI. Cosmological parameters, Astron. Astrophys. 641, A6 (2020).

[2] A. G. Riess, S. Casertano, W. Yuan, L. M. Macri, and D. Scolnic, Large magellanic cloud cepheid standards provide a $1 \%$ foundation for the determination of the Hubble constant and stronger evidence for physics beyond ACDM, Astrophys. J. 876, 85 (2019).

[3] W. L. Freedman et al., The Carnegie-Chicago Hubble program. VIII. An independent determination of the Hubble constant based on the tip of the red giant branch, arXiv:1907.05922.

[4] E. Di Valentino et al., Cosmology intertwined II: The Hubble constant tension, arXiv:2008.11284.

[5] S. Vagnozzi, New physics in light of the $H_{0}$ tension: An alternative view, Phys. Rev. D 102, 023518 (2020).

[6] L. Lancaster, F.-Y. Cyr-Racine, L. Knox, and Z. Pan, A tale of two modes: Neutrino free-streaming in the early universe, J. Cosmol. Astropart. Phys. 07 (2017) 033.

[7] C. D. Kreisch, F.-Y. Cyr-Racine, and O. Doré, Neutrino puzzle: Anomalies, interactions, and cosmological tensions, Phys. Rev. D 101, 123505 (2020).

[8] N. Blinov, K. J. Kelly, G. Z. Krnjaic, and S. D. McDermott, Constraining the Self-Interacting Neutrino Interpretation of the Hubble Tension, Phys. Rev. Lett. 123, 191102 (2019).

[9] K.-F. Lyu, E. Stamou, and L.-T. Wang, Self-interacting neutrinos: Solution to Hubble tension versus experimental constraints, Phys. Rev. D 103, 015004 (2021).

[10] V. Brdar, M. Lindner, S. Vogl, and X.-J. Xu, Revisiting neutrino self-interaction constraints from $Z$ and $\tau$ decays, Phys. Rev. D 101, 115001 (2020).

[11] F. F. Deppisch, L. Graf, W. Rodejohann, and X.-J. Xu, Neutrino self-interactions and double beta decay, Phys. Rev. D 102, 051701 (2020).

[12] T. Brune and H. Päs, Massive Majorons and constraints on the Majoron-neutrino coupling, Phys. Rev. D 99, 096005 (2019).

[13] S. Roy Choudhury, S. Hannestad, and T. Tram, Updated constraints on massive neutrino self-interactions from cosmology in light of the $H_{0}$ tension, J. Cosmol. Astropart. Phys. 03 (2021) 084.

[14] T. Brinckmann, J.H. Chang, and M. LoVerde, Selfinteracting neutrinos, the Hubble parameter tension, and the cosmic microwave background, arXiv:2012.11830.

[15] A. Das and S. Ghosh, Flavor-specific interaction favours strong neutrino self-coupling, arXiv:2011.12315.

[16] A. Mazumdar, S. Mohanty, and P. Parashari, Flavour specific neutrino self-interaction: $H_{0}$ tension and IceCube, arXiv:2011.13685.

[17] M. Archidiacono, S. Gariazzo, C. Giunti, S. Hannestad, and T. Tram, Sterile neutrino self-interactions: $H_{0}$ tension and short-baseline anomalies, J. Cosmol. Astropart. Phys. 12 (2020) 029.

[18] K. J. Kelly, M. Sen, and Y. Zhang, Intimate relationship between sterile neutrino dark matter and $\Delta N_{\text {eff }}$, arXiv:2011 .02487.

[19] H.-J. He, Y.-Z. Ma, and J. Zheng, Resolving Hubble tension by self-interacting neutrinos with dirac seesaw, J. Cosmol. Astropart. Phys. 11 (2020) 003.
[20] M. Berbig, S. Jana, and A. Trautner, The Hubble tension and a renormalizable model of gauged neutrino self-interactions, Phys. Rev. D 102, 115008 (2020).

[21] O. Seto and Y. Toda, Comparing early dark energy and extra radiation solutions to the Hubble tension with BBN, Phys. Rev. D 103, 123501 (2021).

[22] R.-G. Cai, Z.-K. Guo, L. Li, S.-J. Wang, and W.-W. Yu, Chameleon dark energy can resolve the Hubble tension, Phys. Rev. D 103, 121302 (2021).

[23] Y. Gu, M. Khlopov, L. Wu, J. M. Yang, and B. Zhu, Light gravitino dark matter: LHC searches and the Hubble tension, Phys. Rev. D 102, 115005 (2020).

[24] F. Arias-Aragon, E. Fernandez-Martinez, M. GonzalezLopez, and L. Merlo, Neutrino masses and Hubble tension via a majoron in MFV, Eur. Phys. J. C 81, 28 (2021).

[25] E. Grohs, G. M. Fuller, and M. Sen, Consequences of neutrino self interactions for weak decoupling and big bang nucleosynthesis, J. Cosmol. Astropart. Phys. 07 (2020) 001.

[26] M. Escudero and S. J. Witte, A CMB search for the neutrino mass mechanism and its relation to the Hubble tension, Eur. Phys. J. C 80, 294 (2020).

[27] F. Forastieri, M. Lattanzi, and P. Natoli, Cosmological constraints on neutrino self-interactions with a light mediator, Phys. Rev. D 100, 103526 (2019).

[28] E. Di Valentino, O. Mena, S. Pan, L. Visinelli, W. Yang, A. Melchiorri, D. F. Mota, A. G. Riess, and J. Silk, In the realm of the Hubble tension - a review of solutions, arXiv: 2103.01183.

[29] Y. Chikashige, R. N. Mohapatra, and R. D. Peccei, Spontaneously Broken Lepton Number and Cosmological Constraints on the Neutrino Mass Spectrum, Phys. Rev. Lett. 45, 1926 (1980).

[30] Y. Chikashige, R. N. Mohapatra, and R. D. Peccei, Are there real goldstone bosons associated with broken lepton number? Phys. Lett. B 98, 265 (1981).

[31] G. B. Gelmini and M. Roncadelli, Left-handed neutrino mass scale and spontaneously broken lepton number, Phys. Lett. B 99, 411 (1981).

[32] K. Choi and A. Santamaria, 17-KeV neutrino in a singlettriplet majoron model, Phys. Lett. B 267, 504 (1991).

[33] A. Acker, A. Joshipura, and S. Pakvasa, A Neutrino decay model, solar anti-neutrinos and atmospheric neutrinos, Phys. Lett. B 285, 371 (1992).

[34] H. M. Georgi, S. L. Glashow, and S. Nussinov, Unconventional model of neutrino masses, Nucl. Phys. B193, 297 (1981).

[35] J. Schechter and J. W. F. Valle, Neutrino decay and spontaneous violation of lepton number, Phys. Rev. D 25, 774 (1982).

[36] K. Blum, A. Hook, and K. Murase, High energy neutrino telescopes as a probe of the neutrino mass mechanism, arXiv:1408.3799.

[37] G.-y. Huang, T. Ohlsson, and S. Zhou, Observational constraints on secret neutrino interactions from big bang nucleosynthesis, Phys. Rev. D 97, 075009 (2018).

[38] B. Ahlgren, T. Ohlsson, and S. Zhou, Comment on "Is Dark Matter with Long-Range Interactions a Solution to All Small-Scale Problems of $\Lambda$ Cold Dark Matter Cosmology?" Phys. Rev. Lett. 111, 199001 (2013). 
[39] N. Schöneberg, J. Lesgourgues, and D. C. Hooper, The $\mathrm{BAO}+\mathrm{BBN}$ take on the Hubble tension, J. Cosmol. Astropart. Phys. 10 (2019) 029.

[40] J. Venzor, A. Pérez-Lorenzana, and J. De-Santiago, Bounds on neutrino-scalar non-standard interactions from big bang nucleosynthesis, Phys. Rev. D 103, 043534 (2021).

[41] C. Pitrou, A. Coc, J.-P. Uzan, and E. Vangioni, Precision big bang nucleosynthesis with improved Helium-4 predictions, Phys. Rep. 754, 1 (2018).

[42] P. S. Bhupal Dev et al.Neutrino non-standard interactions: A status report, SciPost Phys. Proc. 2, 001 (2019).

[43] E. Aver, K. A. Olive, and E. D. Skillman, The effects of He I $\lambda 10830$ on helium abundance determinations, J. Cosmol. Astropart. Phys. 07 (2015) 011.

[44] R. J. Cooke, M. Pettini, and C. C. Steidel, One percent determination of the primordial deuterium abundance, Astrophys. J. 855, 102 (2018).

[45] A. Arbey, AlterBBN: A program for calculating the $\mathrm{BBN}$ abundances of the elements in alternative cosmologies, Comput. Phys. Commun. 183, 1822 (2012).

[46] A. Arbey, J. Auffinger, K. P. Hickerson, and E. S. Jenssen, AlterBBN v2: A public code for calculating Big-Bang nucleosynthesis constraints in alternative cosmologies, Comput. Phys. Commun. 248, 106982 (2020).

[47] P. A. Zyla et al. (Particle Data Group), Review of particle physics, Prog. Theor. Exp. Phys. (2020), 083C01.

[48] S. Weinberg, Cosmology (Oxford University Press, Oxford, 2008).

[49] J. Froustey and C. Pitrou, Incomplete neutrino decoupling effect on big bang nucleosynthesis, Phys. Rev. D 101, 043524 (2020).

[50] S. Dodelson and M. S. Turner, Nonequilibrium neutrino statistical mechanics in the expanding universe, Phys. Rev. D 46, 3372 (1992).

[51] B. D. Fields, S. Dodelson, and M. S. Turner, Effect of neutrino heating on primordial nucleosynthesis, Phys. Rev. D 47, 4309 (1993).

[52] A. Coc, P. Petitjean, J.-P. Uzan, E. Vangioni, P. Descouvemont, C. Iliadis, and R. Longland, New reaction rates for improved primordial $\mathrm{D} / \mathrm{H}$ calculation and the cosmic evolution of deuterium, Phys. Rev. D 92, 123526 (2015).

[53] Y. Du and J.-H. Yu, Neutrino non-standard interactions meet precision measurements of $N_{\text {eff }}$, arXiv:2101.10475.

[54] S. Hannestad, Structure formation with strongly interacting neutrinos-Implications for the cosmological neutrino mass bound, J. Cosmol. Astropart. Phys. 02 (2005) 011.

[55] S. Hannestad and G. Raffelt, Constraining invisible neutrino decays with the cosmic microwave background, Phys. Rev. D 72, 103514 (2005).

[56] N. F. Bell, E. Pierpaoli, and K. Sigurdson, Cosmological signatures of interacting neutrinos, Phys. Rev. D 73, 063523 (2006).

[57] A. Basboll, O. E. Bjaelde, S. Hannestad, and G. G. Raffelt, Are cosmological neutrinos free-streaming? Phys. Rev. D 79, 043512 (2009).

[58] M. Archidiacono and S. Hannestad, Updated constraints on non-standard neutrino interactions from Planck, J. Cosmol. Astropart. Phys. 07 (2014) 046.
[59] F. Forastieri, M. Lattanzi, and P. Natoli, Constraints on secret neutrino interactions after Planck, J. Cosmol. Astropart. Phys. 07 (2015) 014.

[60] F.-Y. Cyr-Racine and K. Sigurdson, Limits on neutrinoneutrino scattering in the early universe, Phys. Rev. D 90, 123533 (2014).

[61] I. M. Oldengott, C. Rampf, and Y. Y. Y. Wong, Boltzmann hierarchy for interacting neutrinos I: Formalism, J. Cosmol. Astropart. Phys. 04 (2015) 016.

[62] F. Forastieri, M. Lattanzi, G. Mangano, A. Mirizzi, P. Natoli, and N. Saviano, Cosmic microwave background constraints on secret interactions among sterile neutrinos, J. Cosmol. Astropart. Phys. 07 (2017) 038.

[63] I. M. Oldengott, T. Tram, C. Rampf, and Y. Y. Y. Wong, Interacting neutrinos in cosmology: Exact description and constraints, J. Cosmol. Astropart. Phys. 11 (2017) 027.

[64] G. Steigman, Primordial Nucleosynthesis in the precision cosmology era, Annu. Rev. Nucl. Part. Sci. 57, 463 (2007).

[65] M. Bustamante, C. Rosenstrøm, S. Shalgar, and I. Tamborra, Bounds on secret neutrino interactions from high-energy astrophysical neutrinos, Phys. Rev. D 101, 123024 (2020).

[66] S. Shalgar, I. Tamborra, and M. Bustamante, Core-collapse supernovae stymie secret neutrino interactions, arXiv:1912 .09115 [Phys. Rev. D (to be published)].

[67] Y. Farzan, M. Lindner, W. Rodejohann, and X.-J. Xu, Probing neutrino coupling to a light scalar with coherent neutrino scattering, J. High Energy Phys. 05 (2018) 066.

[68] G. Arcadi, J. Heeck, F. Heizmann, S. Mertens, F. S. Queiroz, W. Rodejohann, M. Slezák, and K. Valerius, Tritium beta decay with additional emission of new light bosons, J. High Energy Phys. 01 (2019) 206.

[69] K. C. Y. Ng and J. F. Beacom, Cosmic neutrino cascades from secret neutrino interactions, Phys. Rev. D 90, 065035 (2014).

[70] K. Ioka and K. Murase, IceCube PeV-EeV neutrinos and secret interactions of neutrinos, Prog. Theor. Exp. Phys. (2014), 061E01.

[71] M. Ibe and K. Kaneta, Cosmic neutrino background absorption line in the neutrino spectrum at IceCube, Phys. Rev. D 90, 053011 (2014).

[72] A. Kamada and H.-B. Yu, Coherent propagation of $\mathrm{PeV}$ neutrinos and the Dip in the neutrino spectrum at IceCube, Phys. Rev. D 92, 113004 (2015).

[73] I. M. Shoemaker and K. Murase, Probing BSM neutrino physics with flavor and spectral distortions: Prospects for future high-energy neutrino telescopes, Phys. Rev. D 93, 085004 (2016).

[74] A. DiFranzo and D. Hooper, Searching for MeV-scale gauge bosons with IceCube, Phys. Rev. D 92, 095007 (2015).

[75] A. M. Suliga and I. Tamborra, Astrophysical constraints on non-standard coherent neutrino-nucleus scattering, Phys. Rev. D 103, 083002 (2021).

[76] K. Blum, Y. Nir, and M. Shavit, Neutrinoless double-beta decay with massive scalar emission, Phys. Lett. B 785, 354 (2018).

[77] K. Murase and I. M. Shoemaker, Neutrino Echoes from Multimessenger Transient Sources, Phys. Rev. Lett. 123, 241102 (2019).

[78] G. M. Fuller, R. Mayle, and J. R. Wilson, The Majoron model and stellar collapse, Astrophys. J. 332, 826 (1988). 
[79] Z. G. Berezhiani and A. Y. Smirnov, Matter induced neutrino decay and supernova SN1987A, Phys. Lett. B 220, 279 (1989).

[80] M. Kachelriess, R. Tomas, and J. W. F. Valle, Supernova bounds on Majoron emitting decays of light neutrinos, Phys. Rev. D 62, 023004 (2000).

[81] E. W. Kolb and M. S. Turner, Supernova SN 1987a and the secret interactions of neutrinos, Phys. Rev. D 36, 2895 (1987).

[82] R. V. Konoplich and M. Y. Khlopov, Constraints on triplet Majoron model due to observations of neutrinos from stellar collapse, Sov. J. Nucl. Phys. 47, 565 (1988), https://inis.iaea .org/search/searchsinglerecord.aspx?recordsFor=SingleRecord $\& \mathrm{RN}=20004725$.

[83] Y. Farzan, Bounds on the coupling of the Majoron to light neutrinos from supernova cooling, Phys. Rev. D 67, 073015 (2003).
[84] S. Zhou, Comment on astrophysical consequences of a neutrinophilic 2HDM, Phys. Rev. D 84, 038701 (2011).

[85] L. Heurtier and Y. Zhang, Supernova constraints on massive (Pseudo)Scalar coupling to neutrinos, J. Cosmol. Astropart. Phys. 02 (2017) 042.

[86] J. Bernstein, Kinetic Theory in the Expanding Universe, Cambridge Monographs on Mathematical Physics (Cambridge University Press, Cambridge, England, 1988).

[87] E. Grohs, G. M. Fuller, C. T. Kishimoto, M. W. Paris, and A. Vlasenko, Neutrino energy transport in weak decoupling and big bang nucleosynthesis, Phys. Rev. D 93, 083522 (2016).

[88] S. Hannestad and J. Madsen, Neutrino decoupling in the early universe, Phys. Rev. D 52, 1764 (1995).

[89] A. D. Dolgov, S. H. Hansen, and D. V. Semikoz, Nonequilibrium corrections to the spectra of massless neutrinos in the early universe, Nucl. Phys. B503, 426 (1997). 